\title{
Komplikation nach OP zu spät erkannt?
}

\author{
Ein Gynäkologe wurde aufgrund eines postoperativen Harnstaus \\ verklagt, der zu einer Nierenbeckenentzündung geführt hatte.
}

$\mathrm{N}$ ach einer abdominalen Hysterektomie war vor allem streitig, wann und in welcher Form Schmerzen im Nierenbereich aufgetreten waren, ob und wie die Klägerin solche geschildert hatte und wie reagiert wurde. Während in der Dokumentation erst am zweiten postoperativen Tag „Blähungen“ einmalig notiert waren, behauptete die Klägerin ständige, massive Schmerzen ab dem ersten Tag nach der $\mathrm{OP}$, wovon sie Beklagtem, Schwestern und weiteren Ärzten berichtet habe. Dagegen war für den dritten postoperativen Tag „Pat. hat abgeführt, Bauch jetzt besser" notiert. Am vierten Tag fanden sich keine Notizen zu Beschwerden, am fünften Tag: „Pat. ist nach wie vor empfindlich mit ihrem Bauch; Nierensono gemeldet; 17:00 Uhr: Nierensono gel. -> Blähungen + Aufstau der linken Niere; 20:00 Uhr: Habe Pat. ein Darmrohr angeboten, es wurde abgelehnt“. Die konsiliarisch zugezogene, internistische Oberärztin hatte den V. a. „Aufstau links bei Zustand nach OP und Konkrementen" notiert und empfahl vorerst eine Verlaufskontrolle und Gabe von Sab simplex ${ }^{\oplus}$ oder Paractol'. In der Nacht nahmen allerdings die Schmerzen zu und nach erneuter Sonografie am Folgemorgen, wobei sich die „linke Niere deutlich aufgestaut mit deutlicher $\mathrm{Zu}$ nahme seit gestern abend" zeigte, wurde die Patientin in die Urologie verlegt, wo eine "Stauungspyelonephritis bei distaler Harnleiterenge li.“" diagnostiziert wurde. Es wurden eine Doppel-J-Ureterendoprothese und ein transuretraler Blasenkatheter gelegt, des Weiteren eine Antibiotikatherapie begonnen. Nach fünf Tagen konnte die Klägerin entlassen werden. Ihrer Ansicht nach war es grob fehlerhaft, nicht noch früher reagiert zu haben. Der Verlauf und die Fol- gen, unter anderem streitige Dauerschäden, seien vermeidbar gewesen.

\section{So sah das Gericht den Fall}

Das LG Flensburg (Az. 3 O 300/12) wies die Klage nach Anhörung der Parteien jedoch sachverständig beraten $a b$, da nicht überzeugend feststand, dass der bedauerliche Verlauf auf Fehlverhalten beruhte. Die Verengung des Harnleiters war unstreitig nicht intraoperativ verursacht, sondern laut Sachverständiger durch eine nicht seltene postoperative Ödembildung bedingt. Im Übrigen sei der Spielraum „vom ersten, im Nachhinein verstehbaren Anzeichen über den ersten anzunehmenden Verdacht bis zur Intervention mit Untersuchung und anschließender Behandlung bei einer nicht vermuteten Komplikation im vorliegenden Fall ausgeschöpft, aber nicht überschritten" worden. Ebenso sah dies übrigens die außergerichtliche Gutachterin des MDK. Der Beklagte habe auch alle jeweils gebotenen Untersuchungen ergriffen. Hinsichtlich der mit Blick auf Intensität, Lokalisation und Zeitpunkt streitigen starken Schmerzen in der Nierengegend sei die Klägerin beweisfällig geblieben. Die Kammer konnte allenfalls von anfänglich leichten, generalisierten Schmerzbekundungen ausgehen, die aber selbst wenn sie unmittelbar auftraten, für die OP nicht ungewöhnlich gewesen wären.

Die Klägerin konnte nicht beweisen, dass unmittelbar massive lokale Schmerzen aufgetreten waren. Selbst wenn die Komplikation (noch) früher erkannt worden wäre, stand zudem nicht fest, dass sich etwas am weiteren Verlauf geändert hätte. Das Urteil hielt auch der Berufung beim Schleswig-Holsteinischen OLG stand (Schleswig-Holsteinisches OLG, 4 U 118/15).

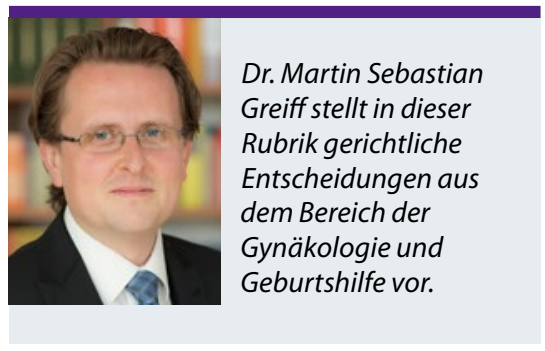

\section{Was bedeutet das Urteil für den klinischen Alltag?}

Während die Gerichte erster Instanz streitige Sachverhalte in tatsächlicher und rechtlicher Sicht zu klären haben, wird in der Berufung die Verhandlung nicht automatisch voll wiederholt. Vielmehr wird, von Ausnahmen abgesehen, in der Regel erst einmal nur geprüft, ob dem ersten Gericht durchgreifende Fehler unterlaufen sind. Das OLG hatte die Entscheidungsfindung vorliegend aber nicht zu beanstanden. Besonders die hinsichtlich $\$ 286 \mathrm{ZPO}$ erfolgte Tatsachensammlung und anschließende Beweiswürdigung sei weder unvollständig noch widersprüchlich gewesen und habe nicht Denk- oder Erfahrungssätze verletzt. Das OLG betonte, dass das Landgericht auch nicht positiv von der fehlerfreien Behandung überzeugt sein musste. Vielmehr habe ausgereicht, dass es nicht ausreichend überzeugt war, dass Fehler vorlagen, insbesondere nicht, dass in dem Umfang und derart konkret Schmerzen beklagt wurden, dass noch frühere Befunderhebungen zwingend gewesen wären. Der Fall zeigt damit einmal mehr, zu wessen Lasten Zweifel bei der Sachverhaltsfeststellung gehen und wie wertvoll eine sorgfältige, im Wesentlichen lückenlose und widerspruchsfreie Dokumentation sein kann.

Dr. Martin Sebastian Greiff, Mag. rer. publ. Fachanwalt für Medizinrecht Partner der Kanzlei Ratzel Rechtsanwälte Romanstraße 77, 80639 München www.ratzel-rechtsanwälte.de 